W.P. Holland

\title{
Snowflakes, snow crystals, hail and rain Nanoparticles in the atmosphere
}

ABSTRACT. Clouds are made up of many low density multimer phase particles. Different clouds have different allotropes of the multimer phase. There are many more allotropes of the multimer phase than there are different types of cloud. A low density multimer particle in a cloud can lose latent heat and become single raindrop. The size of the raindrop indicates the weight of the multimer particle but the latter is a thousand times greater in volume than the raindrop. Although we have seen raindrops these giant multimer particles appear to be invisible! A low density sheetlike multimer particle in a cloud can lose latent heat and split into embryonic snow crystals that combine together to make a snowflake that has a greater volume than its equivalent raindrop though it is smaller than the multimer phase particle from which it was formed. The weight of the raindrop, the snowflake and the multimer will be similar. This study of nanoparticles in the atmosphere reveals that although we can see and photograph the liquid and the solid phases of water as raindrops, hail, snowflakes and snow crystals, we cannot see or photograph any individual and much larger particle of the multimer phase that forms the basis of the many and varied types of cloud.

Nanotechnology Perceptions 10 (2014) 164-172 